\title{
La storia a fumetti
}

L'articolo propone un resoconto della rassegna organizzata dall'Isrec sul linguaggio del fumetto applicato a temi storici. In un dialogo tra autori, insegnanti e storici si è cercato di mettere a fuoco le problematiche che l'arte sequenziale pone alla narrazione della storia, considerando anche il possibile uso didattico delle opere prese in esame.

This article is a report of a series of conferences organized by Isrec in order to analyze a popular medium such as comic books, the way they have dealt with historical issues and the question whether they may represent a reliable source for historians. In a dialogue between authors, teachers and historians, the meetings' aim has been to focus on the questions about the meaning that this language can represent for historians and the teaching of history.

Dai canyon di Tex Willer ai paesaggi caraibici di Corto Maltese, dalla vigilia della Seconda guerra mondiale nelle città dell'Europa orientale agli esotici e orientalizzanti "mondi altri” di Flash Gordon, dal medioevo favolistico di Prince Valiant alla Shoah allegorica di Maus: il fumetto, mass media concepito alla fine dell'Ottocento ma germogliato nel Novecento, ha allargato gli orizzonti geografici e temporali dell'immaginario di milioni di lettori in tutto il globo, usando diverse soluzioni narrative che vanno dalla biografia di personaggi più o meno noti alla vera e propria cronaca storica, per arrivare alla dimensione avventurosa e alla contaminazione con altri generi (horror, sci-fi, erotico ecc.). Attraverso i baloon e le vignette, l'arte sequenziale (così l'ha definita Art Spiegelman) ha creato un sognante canale preferenziale tra la storia e l'immaginazione, dando forma, colore (anche se a volte in bianco e nero...), parole e azione a mondi spesso lontanissimi 
dal nostro. La letteratura disegnata ha scelto spesso come ambientazioni eventi reali, talvolta piegandoli ai bisogni della narrazione, in altri casi rispettando fedelmente il succedersi dei fatti.

Come per cinema e letteratura, questa forma di produzione culturale stringe quindi con il passato un doppio rapporto: interpretazione "storiografica" degli eventi raccontati ma anche fonte storica delle società che tale prodotto ha generato e a cui è destinato. "Storia e fumetto: i linguaggi della storia", un progetto realizzato dall'Istituto storico della Resistenza e dell'età contemporanea di Parma, dall'Associazione culturale L'Abc e da Giulia Bacchini con la collaborazione dell'Associazione culturale Toro e con il patrocinio del Comune di Parma, è nato per indagare attraverso degli incontri pubblici con autori di fumetti e una mostra di tavole originali proprio la relazione tra i comics, la narrazione e la didattica della storia. Il percorso espositivo e le conferenze collegate si proponevano di invitare i partecipanti a interrogarsi su questa complessa relazione, ripensando il fumetto come un "vettore" [Rousso 1987] culturale che avvicina il lettore a "realtà storiche altre", facendosi generatore di immaginario che, tuttavia, va criticamente interrogato come fonte storica legata al contesto socio-culturale che la produce. I tre autori invitati sono stati scelti in base alla qualità delle loro opere e in rapporto al "genere" a cui hanno legato la loro esperienza creativa: Vittorio Giardino, maestro internazionalmente riconosciuto e creatore di alcuni dei più celebrati romanzi a fumetti a tema storico degli ultimi anni (la saga di Max Friedman e Jonas Fink); Paolo Bacilieri, autore di biografie (Sweet Salgari) ma anche di comics seriali per editori come Fandango e Sergio Bonelli; Andrea Ferraris, realizzatore di due graphic novel a sfondo storico (Churubusco, Coconino Press, e La lingua del diavolo, Oblomov) e di un volume di graphic journalism, La cicatrice (Oblomov). I tre ospiti sono stati messi in dialogo con storici, insegnanti della scuola superiore e giovani illustratori per affrontare da punti di vista diversi le relazioni che intercorrono tra storia e fumetto e le possibilità (o le problematiche) che l'uso di questa forma d'arte offre alla didattica della storia.

Nel primo incontro Giardino, in una conversazione con Carlo Ugolotti (Isrec) e Giuseppe Tatalo (Convitto nazionale Maria Luigia), ha messo in luce un nodo fondamentale: mentre la parola scritta non richiede una immediata visualizzazione, essendo un segno "astratto", per il fumetto ogni elemento deve essere presentato visivamente; e quindi nel caso del fumetto storico è preoccupazione dell'autore che ogni cosa sia verosimile e storicamente plausibile. Per esempio, mentre in un romanzo storico o in un saggio lo scrittore può limitarsi a parlare genericamente di "vestiti", per il fumettista tali vestiti devono essere rappresentati e quindi deve cercare fonti che gli dicano come si vestiva la gente nel dato periodo 
e nel dato luogo. Lo stesso discorso vale per la composizione di un menu di un ristorante, o per i cartelloni di propaganda. Il romanziere a fumetti, quindi, se non vuole rinunciare alla verosimiglianza del suo racconto è costretto a trovare fonti dell'epoca che spesso vengono ignorate dallo storico (o se non altro vengono analizzate sotto altre prospettive). Giardino ha quindi raccontato come per preparare la Spagna della guerra civile in cui è ambientato No Pasarán si sia servito di fonti come riviste d'epoca, vecchi menu, mappe stradali, orari della funivia di Barcellona. Nella conclusione della conversazione, il prof. Tatalo ha analizzato rischi e potenzialità dell'utilizzo didattico dei fumetti.

Nell'incontro successivo Paolo Bacilieri, dialogando con Domenico Vitale (Isrec), Andrea Bersellini (Convitto Nazionale Maria Luigia) e il giovane illustratore Federi-

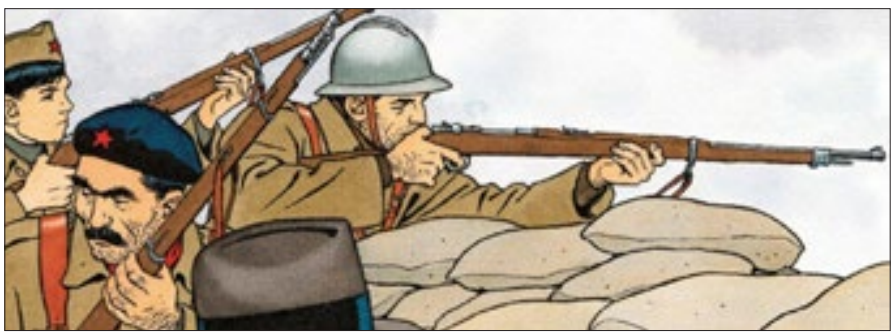

Da Giardino V. 2011, No pasaran: una storia di Max Friedman, Roma: Lizard.

co Febbo (L'Abc),

ha ripreso e analizzato le stesse tematiche introdotte da Giardino (le fonti "storiche" per il fumetto, le fonti di ispirazione per l'autore) attraverso il case study delle sue opere e poi ha allargato il discorso al fumetto in generale, con un particolare focus sull'uso del fumetto seriale come fonte pop per lo studio della storia culturale: personaggi archetipici nella loro invariabilità quali Martin Mystère, Batman ecc. funzionano come barometri dei mutamenti sociali, pur non modificando mai

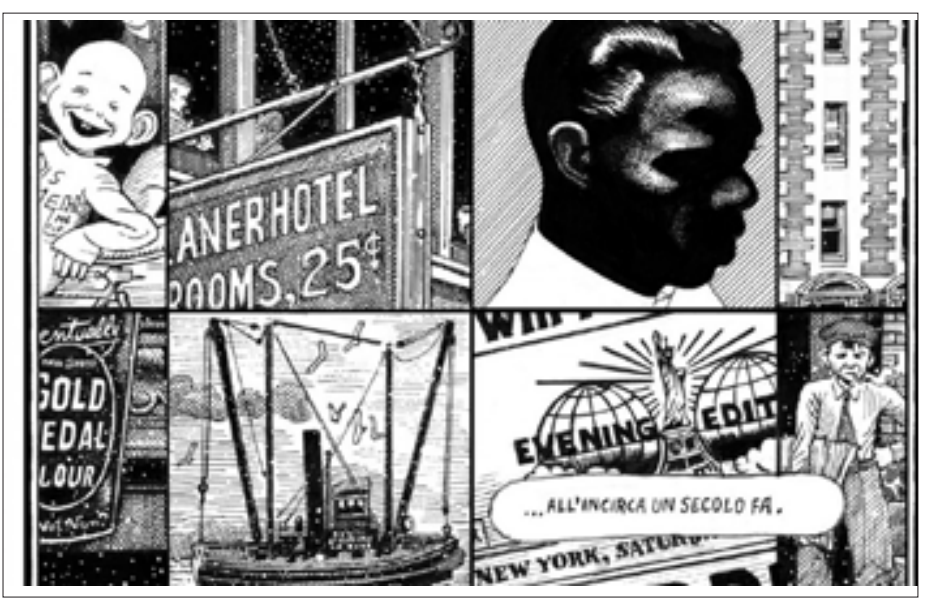

Da Bacilieri P. 2014, Fun, Bologna: Coconino Press.

la struttura narrativa delle avventure delle quali sono protagonisti. Ed è proprio il contrasto della variabilità nella permanenza che permette di rivelare nuovi sviluppi nella sociologia della cultura. L'incontro finale con Andrea Ferra- 
ris, in dialogo con Teresa Malice (Isrec) e Danilo Timelli (Istituto tecnico economico G.B. Bodoni), ha affrontato il tema della costruzione (e decostruzione) dell'immaginario da parte di questo medium (si veda il West di Tex e l'anti-Tex di Churubusco). Si è inoltre tornati sul tema delle fonti e dell'esperienza personale finalizzata al processo creativo (per preparare La cicatrice, reportage sul confine tra Stati Uniti e Messico, l'autore si è fisicamente recato sul luogo). A concludere, ancora una volta, è tornata la questione sull'opportunità di aprire la scuola a strumenti didattici diversi e su come favorire l'alfabetizzazione degli studenti a nuovi linguaggi (cinema, videogiochi, fumetti) che con la scuola stessa si contendono il monopolio della narrazione storica in rapporto alle nuove generazioni.

La mostra invece era divisa in due sezioni: una dedicata alle tavole dei grandi maestri del fumetto, l'altra ai giovani illustratori provenienti da collettivi e associazioni dell'Emilia Romagna. La prima sezione permetteva di constatare, attraver-

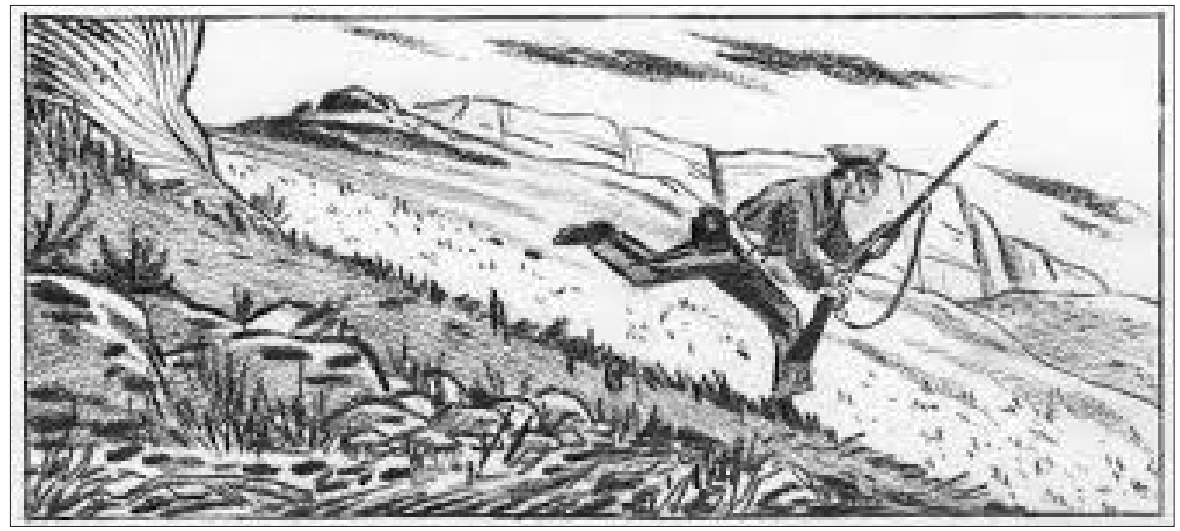

Da Ferraris A. 2015, Churobusco, Bologna: Coconino Press.

so il materiale originale, come maestri riconosciuti del fumetto (tra cui Magnus, Molino e Civitelli, oltre a Giardino e Ferraris) abbiano prestato il loro talento alla ricostruzione di eventi e atmosfere a tema storico. Oltre ad ammirare l'abilità del tratto e la cura nel ricreare mondi lontani dal nostro, al visitatore si chiedeva di interrogare le fonti per riflettere su quanto queste abbiano formato la sua percezione circa l'evento storico preso in considerazione e quanto la lettura di fumetti abbia influito sulla conoscenza e sull'interpretazione dell'evento rappresentato. Nella seconda sezione, autori emergenti (diversi per età e sensibilità rispetto a quelle dei maestri) si sono confrontati con la rappresentazione della storia: giusto per citarne qualcuno si passa dalla Romagna fascista di Silvia Righetti alla Bulgaria anni Venti di Kalina Muhova, dalla Parma devastata dalla guerra di Febbo fino al massacro del Sand Creek immaginato da Mattia Camisa (e probabilmente 
filtrato dall'ascolto dell'omonima canzone di De Andrè). Spazi e tempi lontani e vicini, resi vivi da un medium che rende visibile al fruitore eventi e situazioni "altri", ma che alla sua immaginazione delega di completare le connessioni tra le vignette e di ideare suoni, voci e musiche che costituiscono la colonna sonora della narrazione. Il fumetto ha avvicinato milioni di lettori alla storia, a volte in modo fedele a volte distorcendo il nostro passato in maniera più o meno ingenua; in ogni caso, questa forma d'arte è senza ogni dubbio uno dei grandi generatori di immaginario dell'età contemporanea.

Fu lo storico Marc Ferro a esortare i suoi lettori a considerare l'immaginario come Storia, al pari della "storia" stessa: idee, intenzioni, credenze e immagini possono raggiungere lo stesso grado di incidenza sul tessuto storico sociale di azioni o fatti [Ferro 1980, 101]. Alla stessa conclusione giunse anche E.P. Thompson, che nel suo fondamentale studio sulla classe operaia britannica equiparò l'azione di matrice soggettiva, influenzata dall'immaginario, al motore oggettivo della fattualità: il modo in cui gli attori sociali pensano, amano e sperano ha altrettanta incidenza sulle loro azioni quanto gli agenti esterni [Thompson 1980, 54]. L'immaginario non è quindi relegabile a una figura retorica ma è un motore della storia stessa.

\section{Bibliografia}

Bacilieri P. 2012, Sweet Salgari, Bologna: Coconino Press

Bacilieri P. 2014, Fun, Bologna: Coconino Press

Ferraris A. 2015, Churobusco, Bologna: Coconino Press

Ferraris A. 2017, La cicatrice, Quartu Sant'Elena: Oblomov

Ferraris A. 2018, La lingua del diavolo, Quartu Sant'Elena: Oblomov

Ferro M. 1980, Cinema e storia: linee per una ricerca, Milano: Feltrinelli

Giardino V. 2011, No pasaran: una storia di Max Friedman, Roma: Lizard

Giardino V. 2018, Una vita sospesa: Jonas Fink, Roma: Lizard

Rousso H. 1987, Le syndrome de Vichy, 1944-198..., Paris: Seuil

Spiegelman A. 2004, Maus: A Survivors Tale, New York: Pantheon

Thompson E.P. 1980, The Making of the English Working Class, Harmondsworth: Penguin Books 
